\title{
elsevier_AEP_7850
}

Original article

\section{Working with cancer: health and employment among cancer survivors}

Tainya C. Clarke, $\mathrm{PhD}^{\mathrm{a}}$

Sharon L. Christ, $\mathrm{PhD}$, c

Hosanna Soler-Vila, $\mathrm{PhD}^{\mathrm{a}, \mathrm{d}}$

David J. Lee, $\mathrm{PhD}^{\mathrm{a}}$

Kristopher L. Arheart, EdD

Guillermo Prado, PhD adlease add aditional author:Aberto Caban Martinez, DO, PhD]

Lora E. Fleming, MD, PhD ${ }^{a, e, *}$

L.E.Fleming@Exeter.ac.uk

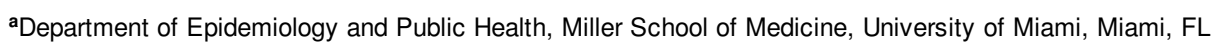

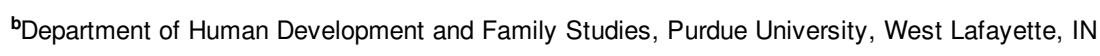

'Department of Statistics, Purdue University, West Lafayette, IN

dDepartment of Preventive Medicine and Public Health, School of Medicine, Universidad Autónoma de Madrid, Madrid, Spain

${ }^{e}$ European Centre for Environment and Human Health, University of Exeter Medical School, Truro, Cornwall, UK

${ }^{*}$ Corresponding author. European Centre for Environment and Human Health, University of Exeter Medical School c/o Knowledge Spa RCHT, Truro, Cornwall TR1 3AE, UK. Tel.: +1-305-243-7842; fax: +1-305-243-5544.

\section{Abstract}

Purpose

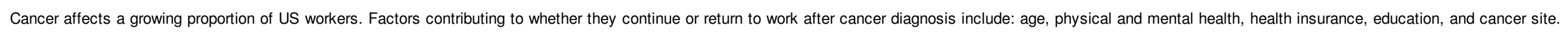
The purpose of this study was to assess the complex relationships between health indicators and employment status for adult cancer survivors.

Methods

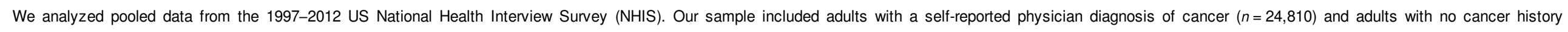

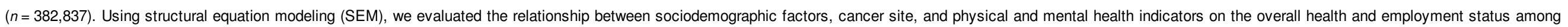
adults with a cancer history

Results

The overall model for cancer survivors fit the data well $\left(\chi^{2}(374)=3654.7, P<\underline{0.001}\right.$ (please make it 0.001 for all $p$ values NOT .001

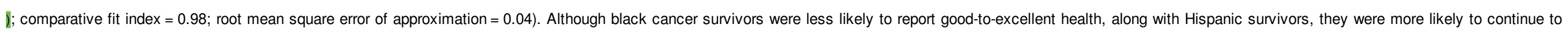

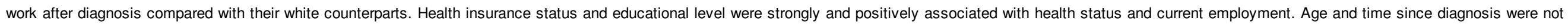
significantly associated with health status or employment, but there were significant differences by cancer site.

Conclusions

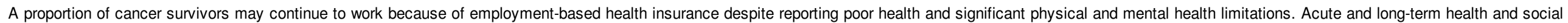


Keywords: Cancer; Survivors; Survivorship; Employment; Epidemiology; Health policy

\section{Introduction}

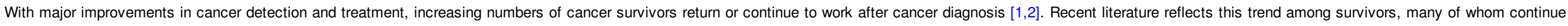

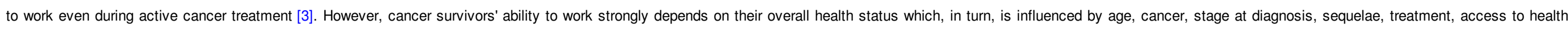
care, and health insurance [4-7].

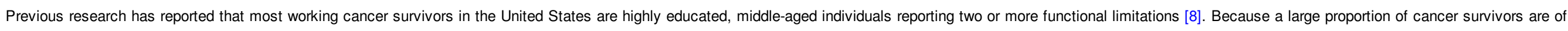

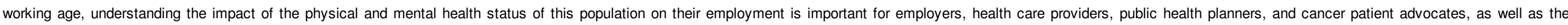

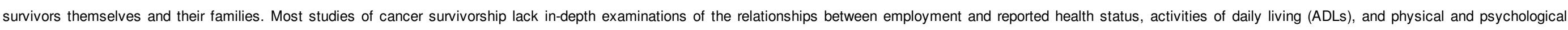

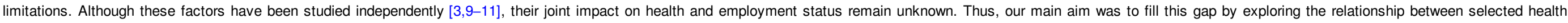
indicators and health and employment status among adult cancer survivors.

\section{Methods}

Participants

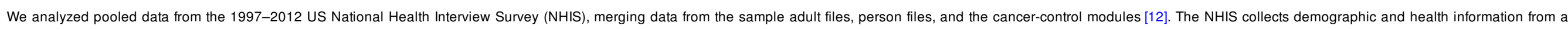

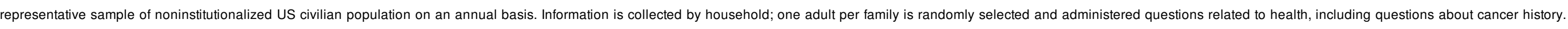
Annual adult response rates to the NHIS data used in this study averaged $71.3 \%$ (range, 69\%-80\%) [13]. All data were self-reported, and participants with missing information were excluded.

\section{Variables}

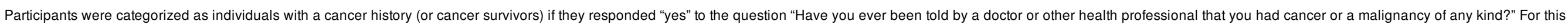

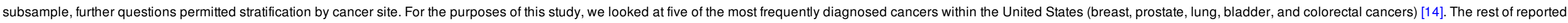
cancer sites were grouped under "other" (referent). Persons with nonmelanoma skin cancer were not included in the study population.

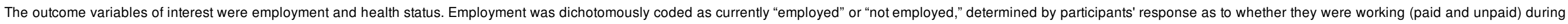

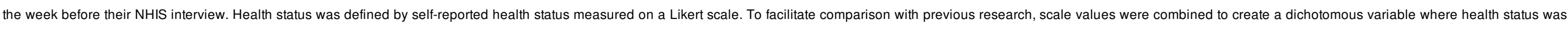
classified as "poor-to-fair" and "good-to-excellent".

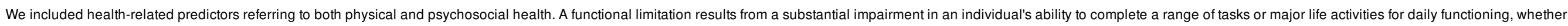

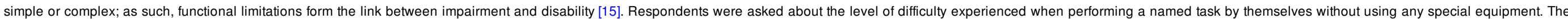

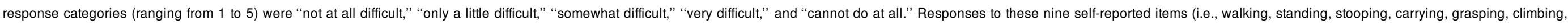

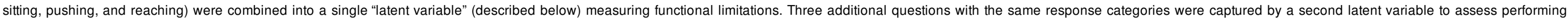
instrumental activities of daily living (IADLs); these are complex skills needed for successful independent living, that is, social functions, relaxing, and shopping [16].

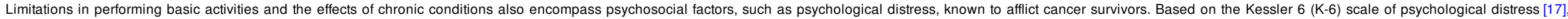

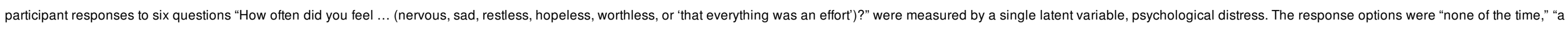
little of the time," "some of the time," "most of the time," and "all of the time being," yielding a score between 1 and 5.

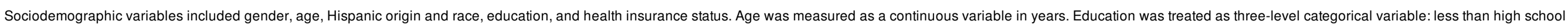




\section{elsevier_AEP_7850}

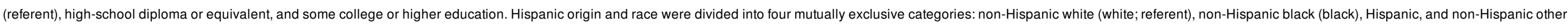

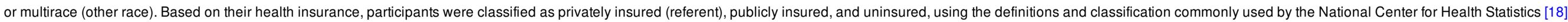

\section{Data analytic plan}

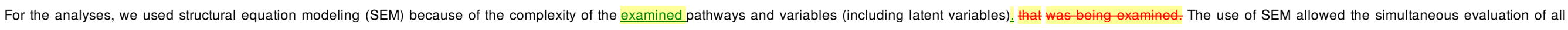

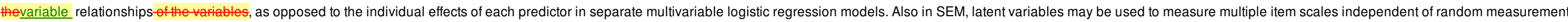

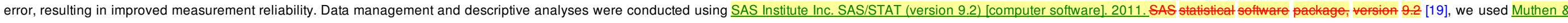

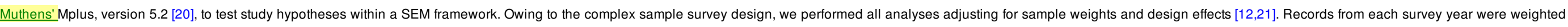

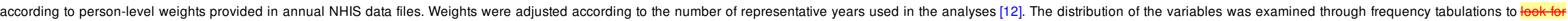
identify outliers and examine the data errors, and variables were tested for collinearity.

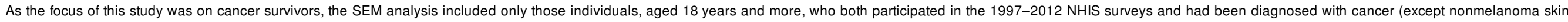

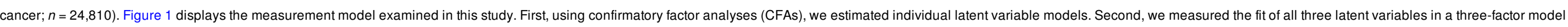
to validate the latent variable measures using model fit indices. Third, we estimated the hypothesized structural equation model.

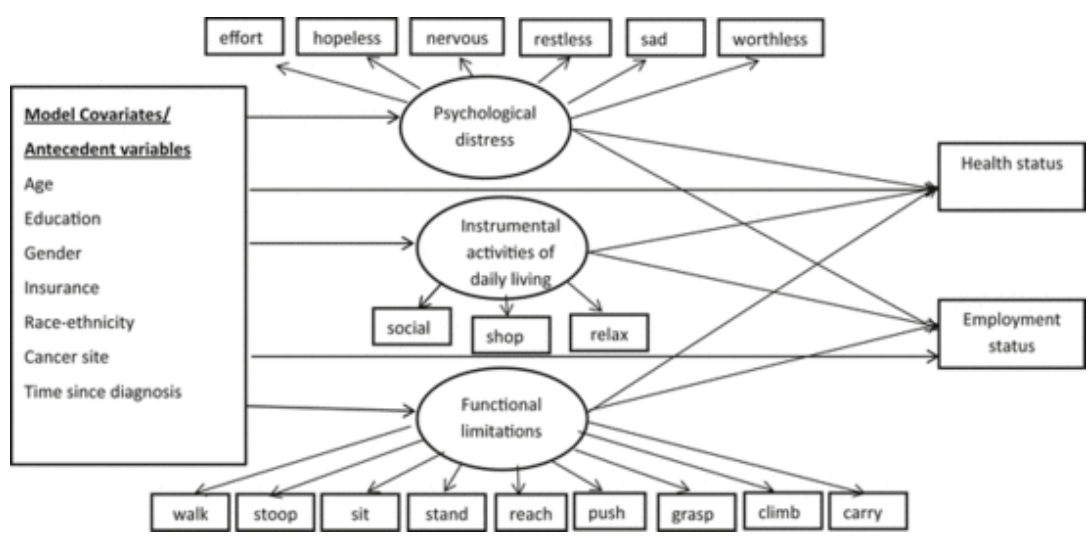

Fig. 1 Measurement model for SEM for adult cancer survivors: National Health Interview Survey 1997-2012.

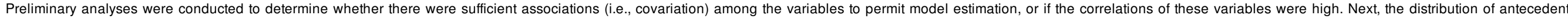

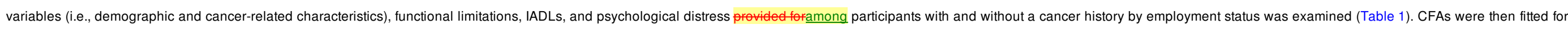
each latent variable separately and together in a three-factor model. Finally, we estimated the hypothesized structural equation model (Fig. 1).

Table 1 A comparison of employment and sociodemographic characteristics of persons in the United States with and without a previous cancer diagnosis: National Health Interview Survey, 1997-2012

\begin{tabular}{|c|c|c|c|c|c|c|c|c|}
\hline \multirow[t]{2}{*}{ Demographic characteristics } & \multicolumn{4}{|c|}{ Persons with cancer diagnosis $n=24,810$} & \multicolumn{4}{|c|}{ Persons without cancer $n=382,837$} \\
\hline & & Not employed* $(n=11,037)$ & & Employed* $(n=13,773)$ & & Employed* $(n=283,767)$ & & ployed $*(n=99,070)$ \\
\hline \multicolumn{9}{|l|}{ Age $(y \underline{r})$} \\
\hline $18-39$ & 3086 & $22.4 \%(20.90-23.90)$ & 947 & $8.6 \%(7.11-10.09)$ & 147,984 & $52.2 \%(50.18-54.22)$ & 35,499 & $35.8 \%(33.79-37.81)$ \\
\hline $40-64$ & 6293 & $45.7 \%(44.98-46.42)$ & 2721 & $24.7 \%(22.31-27.09)$ & 110,865 & $39.0 \%(37.02-40.98)$ & 28,237 & $28.5 \%(26.57-30.43)$ \\
\hline$\geq 65$ & 4394 & $31.9 \%(31.27-32.53)$ & 7369 & $66.7 \%(64.15-69.25)$ & 24,918 & $8.8 \%(6.93-10.67)$ & 35,334 & $35.7 \%(33.48-37.92)$ \\
\hline
\end{tabular}




\section{elsevier_AEP_7850}

\begin{tabular}{|c|c|c|c|c|c|c|c|c|}
\hline Male & 5115 & $37.2 \%(34.41-39.99)$ & 3801 & $34.4 \%(32.53-36.27)$ & 136,429 & $48.1 \%(45.64-50.56)$ & 40,595 & $41.0 \%(38.63-43.37)$ \\
\hline Female & 8658 & $62.8 \%(60.24-65.36)$ & 7236 & $65.6 \%(63.58-67.62)$ & 147,338 & $51.9 \%(48.71-55.09)$ & 58,475 & $59.0 \%(56.56-61.44)$ \\
\hline \multicolumn{9}{|l|}{ Race/ethnicity } \\
\hline Hispanic & 1139 & $8.3 \%(7.36-9.24)$ & 821 & $7.4 \%(6.12-8.68)$ & 52,248 & $18.4 \%(15.98-20.82)$ & 18,853 & $19.0 \%(17.07-20.93)$ \\
\hline Non-Hispanic White & 11,046 & $80.1 \%(76.98-83.22)$ & 8780 & $79.6 \%(74.84-84.36)$ & 177,879 & $62.7 \%(59.47-65.93)$ & 57,908 & $58.5 \%(56.36-60.64)$ \\
\hline Non-Hispanic Black & 1272 & $9.4 \%(9.24-9.56)$ & 1156 & $10.5 \%(9.48-11.52)$ & 41,127 & $14.5 \%(11.27-17.73)$ & 17,005 & $17.1 \%(15.23-18.97)$ \\
\hline Non-Hispanic Other ${ }^{\dagger}$ & 316 & $2.2 \%(2.15-2.25)$ & 280 & $2.5 \%(2.37-2.63)$ & 12,513 & $4.4 \%(3.41-5.39)$ & 5304 & $5.4 \%(4.69-6.11)$ \\
\hline \multicolumn{9}{|l|}{ Education } \\
\hline & 3680 & $29.2 \%(24.90-33.50)$ & 3075 & $29.4 \%(26.17-32.63)$ & 77,367 & $34.0 \%(32.01-35.99)$ & 30,814 & $37.4 \%(35.59-39.21)$ \\
\hline High school/GED & 3437 & $27.3 \%(24.88-29.72)$ & 3344 & $31.9 \%(29.87-33.93)$ & 51,151 & $22.5 \%(20.42-24.58)$ & 23,351 & $28.4 \%(26.53-30.27)$ \\
\hline Some college/higher & 5476 & $43.5 \%(40.26-46.74)$ & 4056 & $38.7 \%(36.67-40.73)$ & 99,024 & $43.5 \%(41.08-45.92)$ & 28,109 & $34.2 \%(32.24-36.16)$ \\
\hline \multicolumn{9}{|l|}{ Health insurance status } \\
\hline Private & 9323 & $70.0 \%(65.99-74.01)$ & 1754 & $16.2 \%(14.23-18.17)$ & 208,918 & $75.9 \%(72.65-79.15)$ & 32,774 & $34.0 \%(32.25-35.75)$ \\
\hline Public & 2490 & $18.7 \%(18.02-19.38)$ & 8488 & $78.4 \%(74.33-82.47)$ & 23,947 & $8.7 \%(6.82-10.58)$ & 49,354 & $51.2 \%(49.16-53.24)$ \\
\hline Uninsured & 1492 & $11.2 \%(8.41-13.99)$ & 584 & $5.4 \%(4.08-6.72)$ & 42,114 & $15.3 \%(13.24-17.36)$ & 14,266 & $14.8 \%(14.32-15.28)$ \\
\hline \multicolumn{9}{|c|}{ Difficulty with instrumental activities of daily living } \\
\hline Yes & 399 & $2.9 \%(2.17-3.63)$ & 671 & $6.1 \%(5.20-7.00)$ & 2783 & $1.0 \%(0.96-1.04)$ & 3599 & $3.6 \%(3.58-3.62)$ \\
\hline No & 13,374 & $97.1 \%(93.36-00.84)$ & 10,366 & $93.9 \%(90.00-97.80)$ & 280,984 & $99.0 \%(95.76-102.2)$ & 95,471 & $96.4 \%(93.92-98.88)$ \\
\hline \multicolumn{9}{|l|}{$\geq 2$ functional limitations } \\
\hline Yes & 4959 & $36.0 \%(32.78-39.22)$ & 6799 & $61.6 \%(58.39-64.81)$ & 11,635 & $4.1 \%(3.58-4.62)$ & 26,055 & $26.3 \%(25.01-27.59)$ \\
\hline No & 8815 & $64.0 \%(60.63-67.37)$ & 4238 & $38.4 \%(35.90-40.90)$ & 272,132 & $95.9 \%(92.06-99.74)$ & 73,015 & $73.7 \%(71.16-76.24)$ \\
\hline \multicolumn{9}{|l|}{ Psychological distress } \\
\hline Yes & 1939 & $14.1 \%(12.30-15.90)$ & 1931 & $17.5 \%(16.23-18.77)$ & 24,809 & $8.7 \%(7.75-9.65)$ & 14,207 & $14.3 \%(12.44-16.16)$ \\
\hline No & 11,874 & $85.9 \%(82.74-89.06)$ & 9106 & $82.5 \%(78.69-86.31)$ & 258,958 & $91.3 \%(87.85-94.75)$ & 84,863 & $85.7 \%(83.22-88.18)$ \\
\hline \multicolumn{9}{|l|}{ Health status } \\
\hline Good-excellent & 11,362 & $82.5 \%(79.62-85.38)$ & 7285 & $66.1 \%(63.59-68.61)$ & 265,173 & $93.5 \%(90.20-96.80)$ & 78,635 & $79.3 \%(77.28-81.32)$ \\
\hline Poor-fair & 2401 & $17.5 \%(15.58-19.42)$ & 3734 & $33.9 \%(31.87-35.93)$ & 18,479 & $6.5 \%(6.37-6.63)$ & 20,366 & $20.7 \%(19.63-21.77)$ \\
\hline \multicolumn{9}{|c|}{ Time since diagnosis of cancer $(\mathrm{y} \underline{\mathrm{r}})$} \\
\hline $0-5$ & 4759 & $42.4 \%(40.30-44.50)$ & 7627 & $39.0 \%(35.75-42.25)$ & - & - & - & - \\
\hline $6-10$ & 2,362 & $21.2 \%(19.10-23.30)$ & 3803 & $20.4 \%(18.00-22.80)$ & - & - & - & - \\
\hline$>10$ & 4076 & $36.4 \%(33.52-39.28)$ & 7976 & $40.6 \%(38.00-43.20)$ & - & - & - & - \\
\hline
\end{tabular}




\begin{tabular}{|c|c|c|c|c|c|c|c|c|}
\hline Bladder & 227 & $2.3 \%(1.74-2.86)$ & 546 & $2.9 \%(2.85-2.95)$ & - & - & - & - \\
\hline Breast & 2189 & $18.3 \%(15.31-21.29)$ & 4386 & $20.9 \%(18.42-23.38)$ & - & - & - & - \\
\hline Colorectal & 605 & $5.4 \%(3.47-7.33)$ & 1885 & $9.4 \%(8.23-10.57)$ & - & - & - & - \\
\hline Lung & 241 & $2.1 \%(0.78-3.42)$ & 881 & $4.5 \%(4.04-4.96)$ & - & - & - & - \\
\hline Prostate & 980 & $9.7 \%(7.79-11.61)$ & 2904 & $16.2 \%(14.35-18.05)$ & - & - & - & - \\
\hline Other cancers $\ddagger$ & 6955 & $62.2 \%(57.54-66.86)$ & 8804 & $46.1 \%(43.56-48.64)$ & - & - & - & - \\
\hline
\end{tabular}

GED = General Education Development certificate.

* Unweighted frequencies, column percentage (95\% confidence interval).

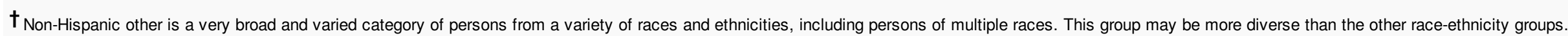

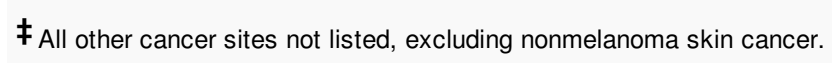

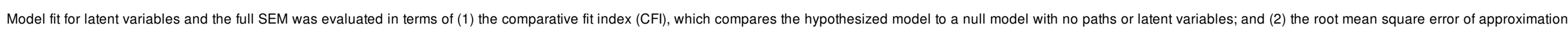

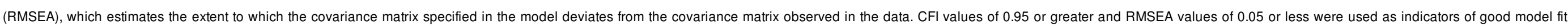

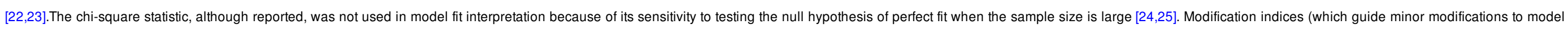

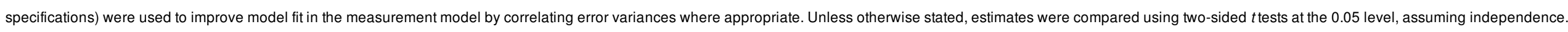

\section{Confirmatory factor analysis}

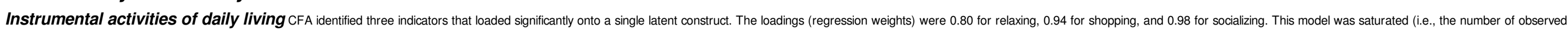
variables was equal to the number of parameters being estimated), therefore model fit statistics are not available.

Both the functional limitations and psychological distress latent variables had good model fit.

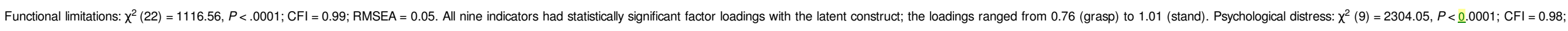
RMSEA $<0.0001$.

All six indicators had statistically significant factor loadings with the latent construct; the loadings ranged from 0.96 (restless) to 1.11 (hopeless).

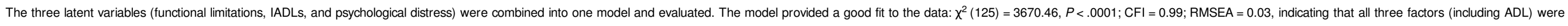
good representations of the data.

\section{Measurement model}

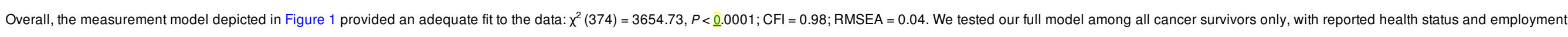
status as the outcomes (Table 2).

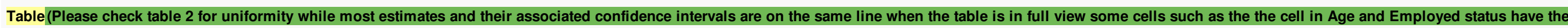

confidence interval on the second line. Can this column be widen to accommodate all numbers on the same line please? Thank you.

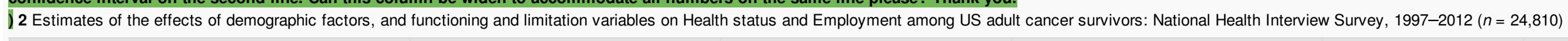

$$
\text { Demographic characteristics }
$$$$
\text { Instrumental activities of daily living }
$$

$$
\begin{gathered}
\text { Functional limitation } \\
\qquad \beta(95 \% \mathrm{Cl})
\end{gathered}
$$

Psychological distress

$\beta(95 \% \mathrm{Cl})$
Health status

OR
Employed status

OR 


\begin{tabular}{|c|c|c|c|c|c|}
\hline Age & $<0.01(0.002-0.002)^{\star * *}$ & $0.02(0.020-0.020)^{\star \star *}$ & $-0.01(-0.010 \text { to } 0.010)^{\star \star \star}$ & $\begin{array}{l}1.0 \text { (If all numbers are } \\
\text { left iustified then the } \\
\text { estimates and } \\
\text { confidence intervals } \\
\text { SHOULD fit on one line } \\
\text { and the editors can } \\
\text { ianore the reauest to } \\
\text { move the confidence } \\
\text { intervals to the line } \\
\text { below. We appreciate } \\
\text { the efforts in making the } \\
\text { tables uniformed. } \\
(0.91-1.13)\end{array}$ & $<1.0(0.94-0.96)^{\star \star \star}$ \\
\hline Male (referent) & 1.00 & 1.00 & 1.00 & - & - \\
\hline Female & $0.03(0.027-0.029)^{\star \star \star}$ & $0.25(0.240-0.260)^{\star \star \star}$ & $0.11(0.104-0.116)^{\star \star \star}$ & $1.3(1.22-1.28)^{\star \star \star}$ & $<1.0(0.88-1.02)$ \\
\hline Race-ethnicity & - & - & - & - & - \\
\hline Non-Hispanic white (referent) & 1.00 & 1.00 & 1.00 & - & - \\
\hline Non-Hispanic black & $0.08(0.079-0.081)^{\star \star \star}$ & $0.37(0.360-0.380)^{\star \star *}$ & $0.07(0.057-0.077)^{\star \star \star}$ & $\begin{array}{l}0.6 \text { (Left justify) } \\
(0.62-0.66)^{\star \star \star}\end{array}$ & $1.1(1.01-1.11)^{\star \star}$ \\
\hline Hispanic & $0.05(0.035-0.055)^{\star \star \star}$ & $0.18(0.166-0.194)^{\star \star \star}$ & $0.12(0.112-0.128)^{\star \star \star}$ & $0.9(0.88-1.02)$ & $1.2(1.11-1.25)^{\star}$ \\
\hline Non-Hispanic other/multirace & $0.04(0.032-0.044)^{\star}$ & $0.12(0.106-0.134)^{\star}$ & $0.09(0.077-0.097)^{*}$ & $\begin{array}{l}0.8 \text { (Should be left } \\
\text { iustified. } \\
{\left[(0.71-0.80)^{\star}\right.}\end{array}$ & $0.9(0.83-0.93)^{\star}$ \\
\hline \multicolumn{6}{|l|}{ Education } \\
\hline Less than high school (referent) & 1.00 & 1.00 & 1.00 & - & - \\
\hline High school/GED & $-0.10(-0.102 \text { to } 0.102)^{\star \star \star}$ & $-0.45(-0.464 \text { to } 0.436)^{\star * \star}$ & $-0.22(-0.226 \text { to }-0.214)^{\star \star \star}$ & $1.6(1.49-1.75)^{\star \star \star}$ & $2.0(1.88-2.04)^{\star \star \star}$ \\
\hline Some college/college degree & $-0.16(-0.157 \text { to } 0.155)^{\star \star \star}$ & $-0.72(-0.739 \text { to } 0.701)^{\star \star \star}$ & $-0.34(-0.340 \text { to }-0.340)^{\star \star \star}$ & $2.2(2.07-2.35)^{\star \star \star}$ & $2.6(2.56-2.70)^{\star \star \star}$ \\
\hline \multicolumn{6}{|l|}{ Health insurance } \\
\hline Private insurance (referent) & 1.00 & 1.00 & 1.00 & - & - \\
\hline Public insurance & $0.05(0.042-0.058)^{\star \star \star}$ & $0.24(0.222-0.258)^{\star \star \star}$ & $0.19(0.187-0.191)^{\star \star \star}$ & $0.5(0.49-0.57)^{\star \star \star}$ & $0.3(0.28-0.32)^{\star \star \star}$ \\
\hline Uninsured & $0.003(0.003-0.003)^{\star \star *}$ & $0.002(0.001-0.003)^{\star \star \star}$ & $0.002(0.002-0.002)^{\star \star \star}$ & $0.9(0.81-0.91)^{\star *}$ & $1.0(0.99-1.03)$ \\
\hline \multicolumn{6}{|l|}{ Cancer history } \\
\hline Time since diagnosis & $<0.01(0.000-0.002)^{\star \star \star}$ & $0.01(0.003-0.011)^{\star \star \star}$ & $<0.01(-0.004 \text { to } 0.008)^{\star \star \star}$ & $1.0(0.91-1.09)$ & $1.0(0.98-1.02)$ \\
\hline Other cancers (referent) & 1.00 & 1.00 & 1.00 & - & - \\
\hline Bladder cancer & $0.02(0.024-0.024)$ & $0.16(0.143-0.173)^{*}$ & $<0.01(0.001-0.008)$ & $1.0(0.87-1.03)$ (ditto) & $1.0(0.85-1.15)$ \\
\hline Breast cancer & $-0.01(-0.009$ to 0.010$)$ & $0.02(0.009-0.037)$ & $-0.07(-0.082 \text { to }-0.058)^{\star \star *}$ & $1.0(0.89-1.11)$ & $1.0(0.82-1.24)$ (ditto) \\
\hline Colorectal cancer & $0.05(0.042-0.058)^{\star \star \star}$ & $0.21(0.196-0.232)^{\star \star \star}$ & $-0.01(-0.014$ to 0.006$)$ & $1.0(0.89-1.09)$ & $0.8(0.70-0.82)^{\star *}$ \\
\hline Lung cancer & $0.17(0.154-0.186)^{\star \star \star}$ & $0.08(0.077-0.081)^{\star \star \star}$ & $0.16(0.159-0.161)^{\star \star \star}$ & $0.8(0.72-0.84)^{\star \star}$ & $0.7(0.69-0.79)^{\star *}$ \\
\hline Prostate cancer & $-0.02(-0.042 \text { to }-0.002)^{\star \star}$ & $-0.12(-0.128 \text { to } 0.112)^{\star \star \star}$ & $-0.08(-0.122 \text { to }-0.118)^{\star \star}$ & $1.1(1.06-1.18)^{\star}$ (ditto) & $0.8(0.69-0.81)^{\star \star \star}$ \\
\hline \multicolumn{6}{|l|}{ Difficulty with functioning and limitations } \\
\hline Instrumental activities of daily living & - & - & - & $0.9(0.79-0.91)^{\star \star \star}$ & $0.8(0.75-0.83)^{\star \star \star}$ \\
\hline
\end{tabular}




\section{elsevier_AEP_7850}

Functional limitation

Psychological distress

Health status

${ }^{\star} P<\underline{0} .05,{ }^{* \star} P<\underline{0} .01,{ }^{* \star *} P<\underline{0} .001$

$95 \%$ Cls presented for beta values and ORs.

These analyses of anonymized data were approved by the University of Miami School of Medicine Human Subjects Committee.

\section{Results}

\section{Cancer survivors versus individuals without a cancer history}

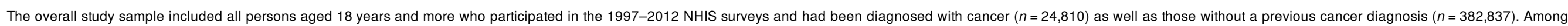
those currently working, 13,773 were cancer survivors, and 283,767 were adults without a cancer history (Table 1).

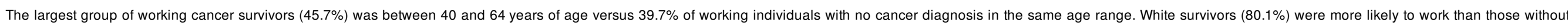

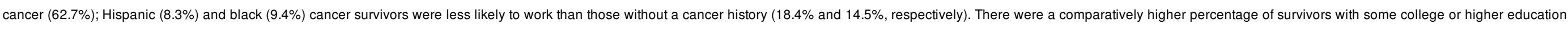

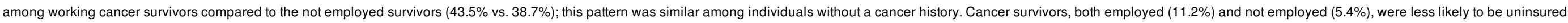
compared to those without cancer whether employed (15.3\%) or not employed (14.8\%).

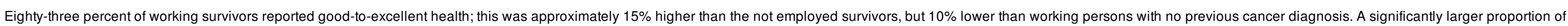

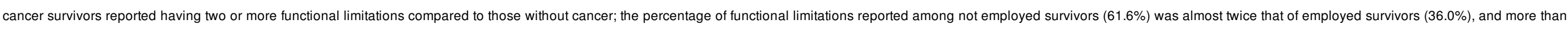

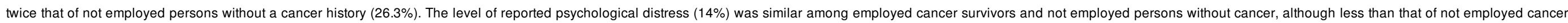
survivors $(17 \%)$.

\section{Cancer survivors only}

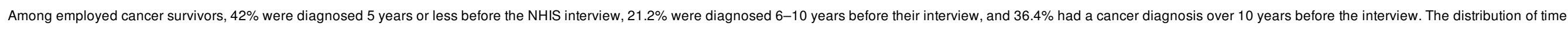

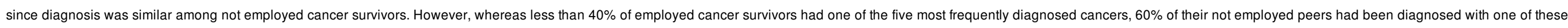
cancers.

\section{Health status}

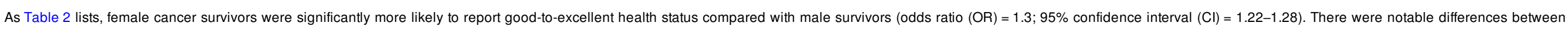

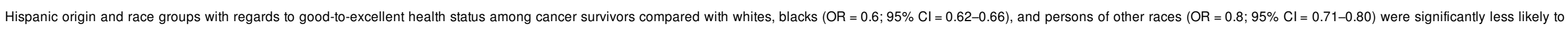

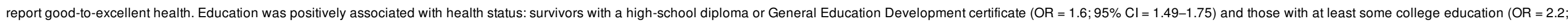

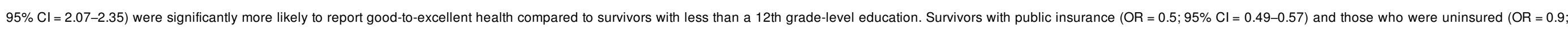

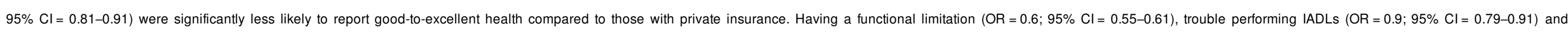
psychological distress $(\mathrm{OR}=0.5 ; 95 \% \mathrm{Cl}=0.50-0.58)$ were all significantly inversely related to health status.

\section{Employment status}

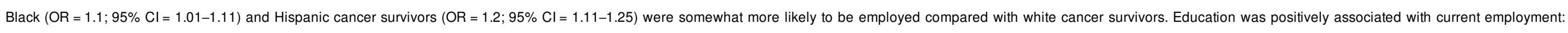

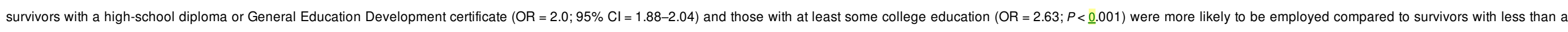

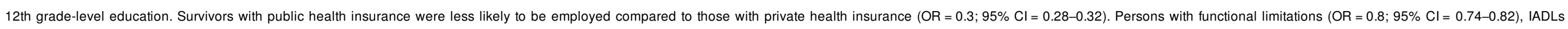




\section{elsevier_AEP_7850}

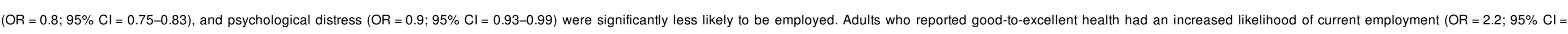
2.21-2.25) compared with those who reported poor-to-fair health.

\section{Cancer history}

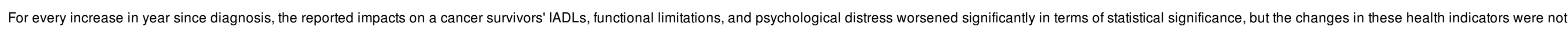

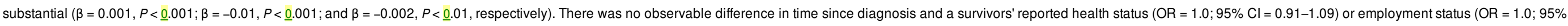
$\mathrm{Cl}=0.98-1.02 ;$ Table 2).

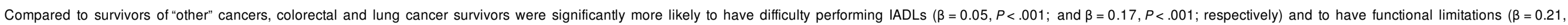

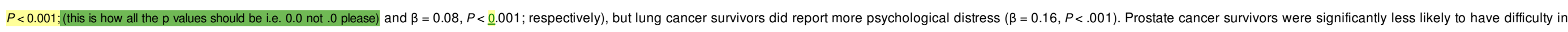

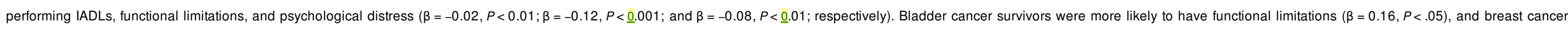
survivors were significantly less likely to report psychological distress $(\beta=-0.07, P<\underline{0} .001)$.

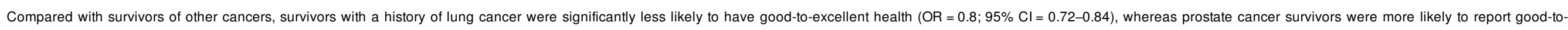

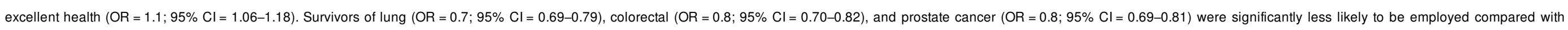
survivors of other cancers (Table 2).

\section{Discussion}

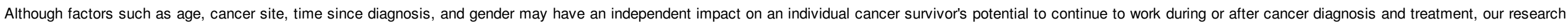

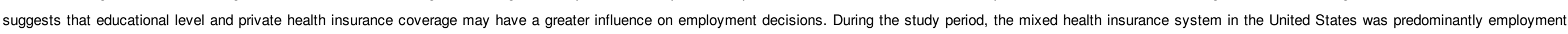

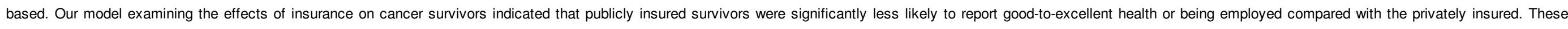

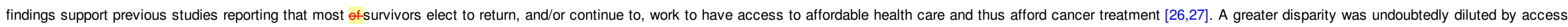
to public insurance, such as Medicaid among the older and qualified disabled cancer survivors [28].

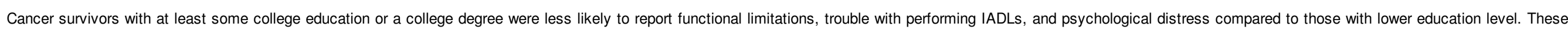

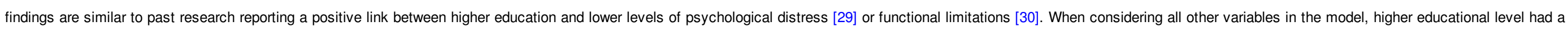

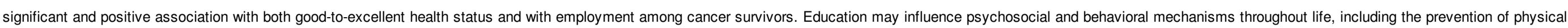

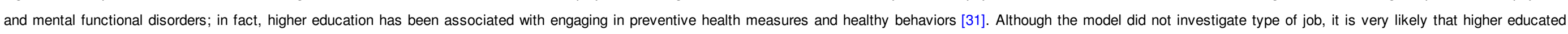

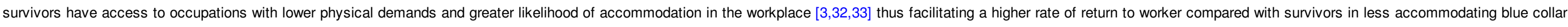
jobs.

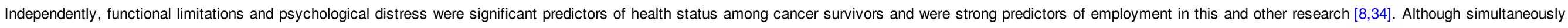

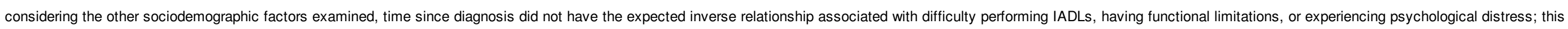
may be due to the fact that there was non participation in the NHIS by those hospitalized or very sick and who had recently died as a result of the disease.

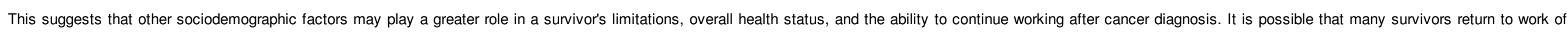

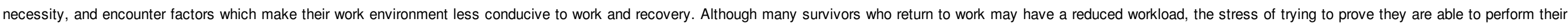

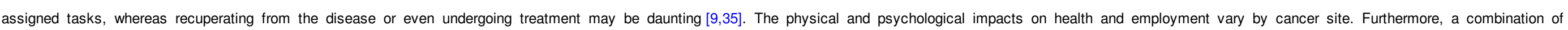
sociodemographic factors (e.g. education) may increase or decrease the negative associations between type of cancer (e.g. lung cancer) and reported health status or current employment for different individuals.

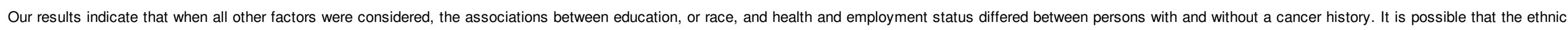

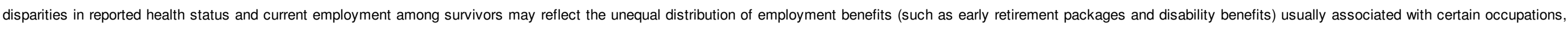

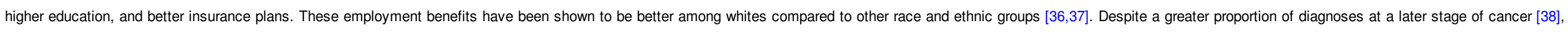




\section{elsevier_AEP_7850}

black and Hispanic cancer survivors are as likely to remain in the workforce as their counterparts with no cancer diagnosis despite their reports of poorer health compared to whites.

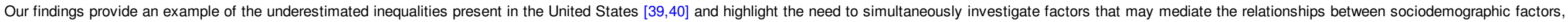

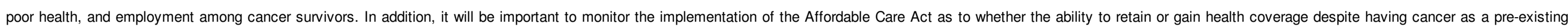
conditions is associated with an increase in the number of lower income Americans who can afford insurance coverage and thus return to work for reasons other than an insurance-based access to care.

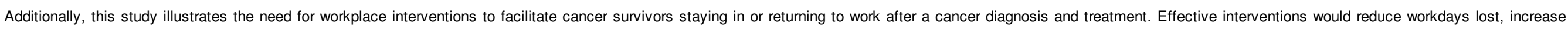
productivity, and reduce psychosocial stresses associated with unemployment and treatment costs among cancer survivors.

\section{Limitations}

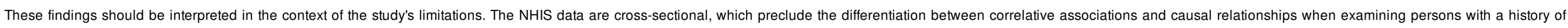

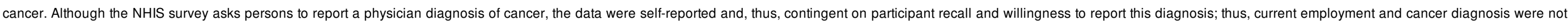
validated. Important clinical variables (such as stage at diagnosis or recurrences of cancer) were also not available; and type of treatment received was made available only in the $2010 \mathrm{NHIS}$ cancer-control module.

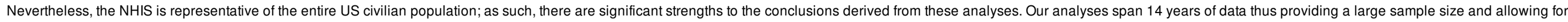

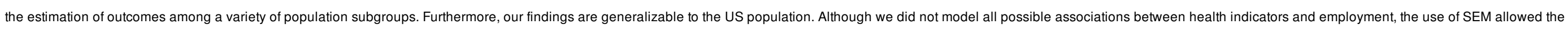
simultaneous evaluation of all the relationships of the variables completely and simultaneously.

\section{Conclusions}

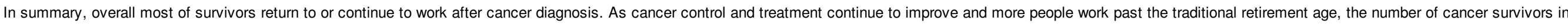

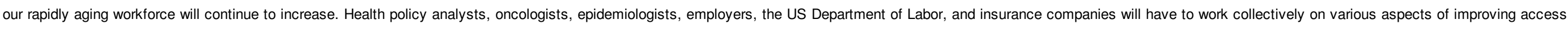

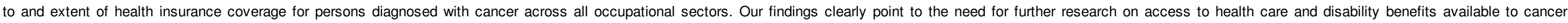

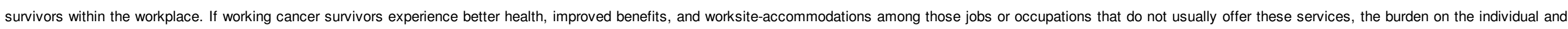
the health care system would be reduced as more survivors would have the ability to return to work or to continue to work throughout their treatment.

\section{Acknowledgments}

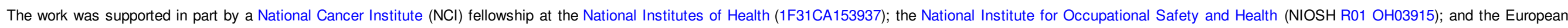
Regional Development Fund Programme and European Social Fund Convergence Programme for Cornwall and the Isles of Scilly (the University of Exeter Medical School).

\section{References}

[1]

B. Hoffman, Cancer survivors at work: a generation of progress, CA Cancer J Clin 55, 2005, 271-280.

[2]

Horner MJ, Ries LAG, Krapcho M, Neyman N, Aminou R, Howlander N, et al. National Cancer Institute. SEER Cancer Statistics Review, 1975-2006.

[3]

Z. Amir and J. Brocky, Cancer survivorship and employment: epidemiology, Occup Med (Lond) 59 (6), 2009, 373-377.

[4]

E.R. Spelten, M.A. Sprangers and J.H. Verbeek, Factors reported to influence the return to work of cancer survivors: a literature review, Psychooncology 11, $2002,124-131$.

[5]

P. Short, J.J. Vasey and K. Tuncelli, Employment pathways in a large cohort of adult cancer survivors, Cancer 103, 2005, 1292-1301. 


\section{elsevier_AEP_7850}

[6]

M. Hewitt, J.H. Rowland and R. Yancik, Cancer survivors in the United States: age, health, and disability, J Gerontol A Biol Sci Med Sci 58, $2003,82-91$.

[7]

R.R. Bouknight, C.J. Bradley and Z. Luo, Correlates of return to work for breast cancer survivors, J Clin Oncol 24 (3), 2006, 345-353.

[8]

J.H. Rowland and R. Yancik, Cancer survivorship: the interface of aging, comorbidity, and quality care, J Natl Cancer Inst 98, 2006, 504-505.

[9]

S. Dellapasqua, M. Colleoni and A. Goldhirsch, Focusing on physical function limitations in elderly women surviving cancer: any opportunity for improvement?, Nat Clin Pract Oncol 3, 2006, 598-599.

[10]

C. Sweeney, K.H. Schmitz, D. Lazovich, B.A. Virnig, R.B. Wallace and A.R. Folsom, Functional limitations in elderly female cancer survivors, J Natl Cancer Inst 98 (8), $2006,521-529$.

[11]

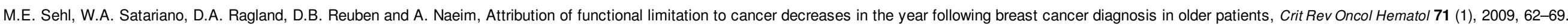

[12]

S.L. Botman, T.F. Moore, C.L. Moriarity and V.L. Parsons, Design and estimation for the National Health Interview Survey, 1995-2004, National Center for Health Statistics Vital Health Stat 2 (130), 2000.

[13]

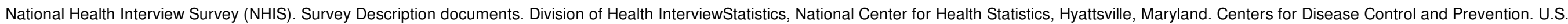
Department of Health and Human Services. http://www.cdc.gov/nchs/nhis/quest_data_related_1997_forward.htm [accessed 19.7.2012].

[14]

American Cancer Society, Cancer Facts \& Figures 2012, 2012, American Cancer Society; Atlanta.

[15]

L.M. Verbrugge and A.M. Jette, The disablement process, Soc Sci Med 38, 1994, 1-14.

[16]

National Cancer Institute. Dictionary of Cancer terms. http://www.cancer.gov/dictionary?cdrid=430402 [accessed 6.6.2011].

[17]

R.C. Kessler, G. Andrews, D. Mroczek, T.B. Ustun and H.U. Wittchen, The World Health Organization Composite International Diagnostic Interview Short-Form (CIDI-SF), Int J Methods Psychiatr Res 7, 1998, 171-185.

[18]

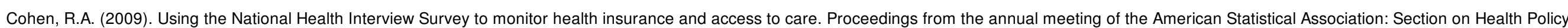
statistics. JSM. 2009; 108-118.

[19]

SAS Institute. Survey data analysis. http://support.sas.com/documentation/cdl/en/statug/66859/HTML/default/viewer.htm\#statug_introsamp_sect019.htm [accessed 10.11.2014].

[20]

B. Muthén and L. Muthén, Mplus user's guide, 6 th ed., 1998-2010, Muthén \& Muthén; Los Angeles, CA. 


\section{elsevier_AEP_7850}

[21]

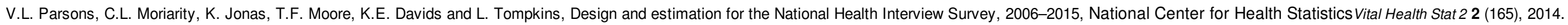

\section{[22]}

R.B. Kline, Principles and practice of structural equation modeling, 3rd ed., 2010, Guilford Press; New York.

[23]

D.A. Kenny, Assessing model fit, $2010 \mathrm{http}: / /$ davidakenny.net/cm/fit.html, [accessed 23.9.2010].

[24]

P.M. Bentler and D.C. Bonnet, Significance tests and goodness of fit in the analysis of covariance structures, Psychol Bull 88 (3), 1980, 588-606.

[25]

K. Jöreskog and D. Sörbom, LISREL 8: structural equation modeling with the SIMPLIS command language, 1993, Scientific Software International Inc; Chicago, IL.

[26]

C.J. Bradley, D. Neumark, Z. Luo and H.L. Bednarek, Employment-contingent health insurance, illness, and labor supply of women: evidence from married women with breast cancer, Health Econ 16, 2007 , 19-37.

[27]

E.A. Finkelstein, F.K. Tangka, J.G. Trogdon, S.A. Sabatino and L.C. Richardson, The personal financial burden of cancer for the working age population, Am J Manag Care 15, 2011 , 801-806.

[28]

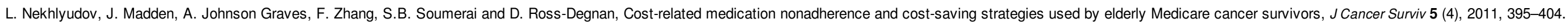

[29]

A. Brännlund and A. Hammarström, Higher education and psychological distress: a 27-year prospective cohort study in Sweden, Scand J Public Health 42 (2), 2014, 155-162.

[30]

Z. Zimmer and J.S. House, Education, income, and functional limitation transitions among American adults: contrasting onset and progression, Int J Epidemio/ 32, 2003, $1089-1097$.

[31]

J.M. Fletcher and D.E. Frisvold, Higher education and health investments: does more schooling affect preventive health care use?, J Hum Cap 3 (2), 2009, 144-176.

[32]

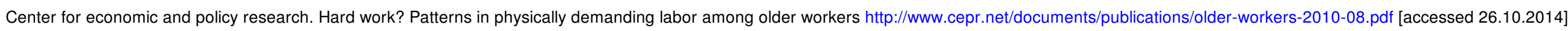

[33]

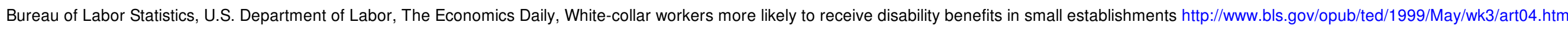
[accessed 26.10.2014].

[34]

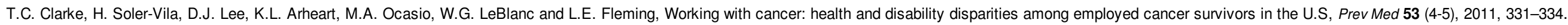

[35]

G. Pransky, Work and cancer survivors, J Occup Environ Med 52 (1), 2010, 111.

[36]

U. S. Bureau of Labor Statistics, Labor force characteristics by race and ethnicity, 2008, 2009, U.S. Department of Labor; Washington, DC http://www.bls.gov/cps/cpsrace2009.pdf, [accesseed 23.9.2010]. 


\section{elsevier_AEP_7850}

\section{[37]}

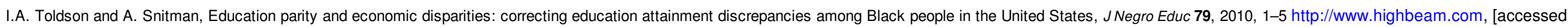
10.9.2011].

[38]

H. Soler-Vila, S.V. Kasl and B.A. Jones, Prognostic significance of psychosocial factors in African-American and white breast cancer patients: a population-based study, Cancer 98 (6), $2003,1299-1308$.

\section{[39]}

W.J. Wilson, The Truly disadvantaged: the inner city, the underclass, and public policy, 1987, University of Chicago Press; Chicago.

\section{[40]}

W.J. Wilson, Social theory and the concept 'underclass', In: D.B. Grusky and R. Kanbur, (Eds.), Poverty and inequality, 2006, Stanford University Press; Stanford.

\section{Queries and Answers}

Query: Please note that the abstract subhead "Background" has been changed to "Purpose" as per journal style specifications.

Answer: Thank you, that is okay.

Query: Please provide manufacturer details for "SAS package version 9.2" software and "Mplus version 5.2" software in the sentence "Data management and..."

Answer: We are not sure what you mean but have attempted to update the document withe information required. However we usually provide the text as was written previously.

Query: Please provide grant number for "National Cancer Institute" if any.

Answer: This has been provided in parentheses following National Cancer Institute ( $\mathrm{NCl})$ of the National Institutes of Health........

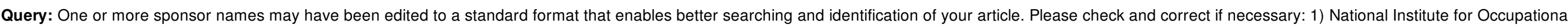
Safety and Health, United States.

Answer: That is ok, thank you.

Query: Please provide an appropriate first column head for both the Tables and also check the edit to the value corresponding to of table entry "Employed" and amend if necessary. Answer: We have added the heading "Demographic characteristics" to both Tables 1 and 2.

Query: Please provide publisher name and location details for the Refs. 2 and 18.

Answer: The publisher is the Surveillance, Epidemiology and End Results (SEER) Program, National Cancer Institute (NCl) (NCl) http://seer.cancer.gov/archive/csr/1975_2006/

Query: Please provide the year of publication for the Ref. 13.

Answer: 2014

Query: Please confirm that given names and surnames have been identified correctly.

Answer: These are correct thank you 